\title{
Alteration of Collagen Phenotypes in Ischemic Cardiomyopathy
}

\author{
Debabrata Mukherjee, and Subha Sen \\ Department of Heart and Hypertension Research, The Cleveland Clinic Foundation, Cleveland, Ohio 44195
}

\begin{abstract}
Ischemic cardiomyopathy refers to a significant impairment of left ventricular function, a condition resulting from atherosclerotic coronary artery disease. The left ventricular ejection fraction is usually $35 \%$ or less, and electron microscopy shows an increased deposition of collagen in the space between the capillaries and the myocytes. The present study shows the alteration in collagen concentration and phenotypes in ischemic cardiomyopathy, and the effect captopril treatment has on these parameters. In patients with ischemic cardiomyopathy, collagen concentration estimated from hydroxyproline increased from $7.96 \pm 1.24 \mathrm{mg} / \mathrm{g}$ to $13.97 \pm 1.30 \mathrm{mg} / \mathrm{g}, P<0.05$. Ischemic cardiomyopathic patients given captopril therapy had a signifcantly lower collagen concentration of $10.03 \pm 1.46 \mathrm{mg} / \mathrm{g}, P$ $<0.05$. The collagen type I:III ratio decreased from $1.93 \pm 0.52$ to $1.23 \pm 0.27$ in patients with ischemic cardiomyopathy. Of these patients, those receiving captopril had a collagen type I:III ratio of $1.49 \pm 0.38$, which did not differ significantly from the ratio of individuals with normal myocardium. There was no significant difference in type I collagen concentration in the myocardium of normal individuals, patients with ischemic cardiomyopathy, and patients with ischemic cardiomyopathy receiving captopril therapy. The type III collagen concentration increased significantly from $2.56 \pm 0.21 \mathrm{mg} / \mathrm{g}$ in normal myocardium to $6.10 \pm 0.58 \mathrm{mg} / \mathrm{g}$ in ischemic cardiomyopathic myocardium. Patients receiving captopril had a myocardial collagen type III concentration of $4.87 \pm 0.64 \mathrm{mg} / \mathrm{g}$, which was significantly lower than that found in patients with ischemic cardiomyopathy. An increased deposition of type III collagen may be partly responsible for altering the compliance of the myocardium, resulting in dilatation of the heart and possibly leading to eventual heart failure. (J. Clin. Invest. 1991. 88:1141-1146.) Key words: captopril • collagen types I and III • collagen concentration • ejection fraction
\end{abstract}

\section{Introduction}

The term "ischemic cardiomyopathy," popularized by Burch and co-workers $(1,2)$, refers to severe myocardial dysfunction that may result from occlusive coronary artery disease. The pathology of ischemic cardiomyopathy reflects the diffuse nature of myocardial involvement. Electron microscopy reveals an increased deposition of collagen between the capillaries and

Address correspondence to Subha Sen, Ph.D., Dept. of Heart \& Hypertension Research, Research Institute FF-3, The Cleveland Clinic Foundation, 9500 Euclid Ave., Cleveland, OH 44195-5071.

Received for publication 7 January 1991 and in revised form 10 June 1991.

J. Clin. Invest.

(c) The American Society for Clinical Investigation, Inc.

0021-9738/91/10/1141/06 $\$ 2.00$

Volume 88, October 1991, 1141-1146 myocytes (3). It is now accepted that the myocardium contains a collagen matrix, consisting primarily of type I and type III collagens (4), which is a major determinant of myocardial architecture, structural integrity, and mechanical properties (5). Although to date, the alteration of collagen content and collagen phenotypes in ischemic cardiomyopathy has not been similarly studied. Our present study deals with the alteration of collagen concentration and the alteration from collagen type I to type III deposition in ischemic cardiomyopathy. In spontaneously hypertensive rats (SHR) ${ }^{1}$ we have recently shown (6) that collagen phenotypic distribution became altered during the evolution of hypertensive hypertrophy and that this alteration could be ameliorated by captopril therapy. Because captopril results in long-term hemodynamic and symptomatic improvement and has been shown to prolong survival in humans $(7,8)$, we studied the effects of captopril treatment on the collagen concentration and alteration in collagen type I and type III deposition in myocardial tissues in patients with ischemic cardiomyopathy given this therapy before transplantation. Very little work has been done on the alterations of collagen resulting from pathologies in the human heart, although Epstein et al. (9) reported the presence of both type I and type III collagen in postinfarction scar. Using immunohistochemistry, Shekhonin et al. (10) have shown that in postinfarction cardiosclerosis type III collagen is diffusely distributed, but type I is restricted to the deeper regions of fibrous tissue. The present study is the first of this type to demonstrate the changes in collagen metabolism in ischemic cardiomyopathy.

\section{Methods}

\section{Study groups}

At The Cleveland Clinic Foundation, about 125 heart transplants are performed each year. All heart tissue used in this study was obtained through the Foundation's transplant program. This study consisted of 20 patients divided into three groups. The first group contained six individuals, five males and one female aged 17-58 yr, whose mean age was $32.00 \pm 16.40 \mathrm{yr}$. These individuals died of noncardiovascular causes, and their hearts were designated normal on gross examination and histopathology. The second group was composed of seven male patients with ischemic cardiomyopathy aged 43-54 yr, whose mean age was $49.71 \pm 3.49 \mathrm{yr}$. Before transplantation, these patients had been treated with drugs other than angiotensin converting enzyme inhibitors. The third group, six males and one female aged 45-63 yr, whose mean age was $54.28 \pm 7.15 \mathrm{yr}$, were ischemic cardiomyopathic patients who had been receiving captopril for a duration of 6-26 mo. These patients received captopril at a dose of $78.50 \pm 33.60 \mathrm{mg}$ for a duration of $2.22 \pm 0.27 \mathrm{yr}$. Their diagnoses of ischemic cardiomyopathy were based on clinical and pathological examination.

\section{Collection of tissue}

The explanted heart was immediately immersed in cold cardioplegic solution, made by mixing $20 \mathrm{ml}$ of cardioplegic concentrate $(\mathrm{KCl}$,

1. Abbreviation used in this paper: SHR, spontaneously hypertensive rat. 
$1.193 \mathrm{~g} ; \mathrm{MgCl}_{2}, 3.253 \mathrm{~g} / 20 \mathrm{ml}$ ) with $10 \mathrm{ml}$ sodium bicarbonate (1 $\mathrm{mEq} / \mathrm{ml})$ and $1,000 \mathrm{ml}$ of Ringers lactate solution $(86 \mathrm{mg} \mathrm{NaCl}, 30 \mathrm{mg}$ $\mathrm{KCl}, 33 \mathrm{mg} \mathrm{CaCl} / 100 \mathrm{ml}$ ). The heart was subsequently weighed and inspected, and pieces were removed from various sites for histopathological studies. For our studies we removed a $500-\mathrm{mg}$ piece of tissue from the left ventricular apex, avoiding the infarcted area of the myocardium.

\section{Determination of hydroxyproline}

Hydroxyproline was determined by using a modified Stagemann procedure (11). Myocardial tissue was homogenized, and hydrolysis of the sample solution was done with $6 \mathrm{~N} \mathrm{HCl}$ at $100^{\circ} \mathrm{C}$ for $24 \mathrm{~h}$. The hydrolyzed samples were dried using a flash evaporator. Hydroxyproline standard solutions of $2,4,6,8$, and $10 \mu \mathrm{g} / \mathrm{ml}$ were made. A reagent blank was included in the procedure by substituting water for the hydroxyproline solution; the absorbance was corrected accordingly. 0.5 $\mathrm{ml}$ of hydroxyproline standard solutions of different strengths and homogenates of heart samples were taken in glass tubes, and $1 \mathrm{ml}$ of isopropanol was added to each. The tubes were then vortexed. To this solution, $0.5 \mathrm{ml}$ of oxidant $(0.35 \mathrm{~g}$ chloramine $\mathrm{T}+5 \mathrm{ml}$ water $+20 \mathrm{ml}$ citrate buffer) was added, vortexed, and allowed to stand for $4 \mathrm{~min}$. $3.25 \mathrm{ml}$ of Ehrlich reagent ( $3 \mathrm{ml}$ Ehrlich $+16 \mathrm{ml}$ isopropanol) was subsequently added. After the tubes were kept at $25^{\circ} \mathrm{C}$ for $18 \mathrm{~h}$, the intensity of red coloration was measured at wavelength $558 \mathrm{~nm}$ using a model DU50 spectrophotometer (Beckman Instruments, Inc., Fullerton, CA). The amount of hydroxyproline in the unknown samples was calculated using the standard curve. The collagen content was estimated by multiplying the hydroxyproline content by a factor of 8.2 .

\section{Quantitation and typing of collagen}

Extraction of collagen. Reference grade collagen standards were obtained from Calbiochem-Behring Corp., San Diego, CA. Experiments were performed on left ventricular tissue from patients undergoing transplantation. The extraction and phenotyping of the collagen was performed using the procedure described by Laurent et al. (12). In this method, the SDS was shown to remove the bulk of noncollagen proteins, leaving an insoluble residue which could then be reacted with cyanogen bromide without further purification. Furthermore, cyanogen bromide was shown to solubilize essentially all the collagen in the residue, leaving an insoluble pellet whose amino acid analysis has been shown by Laurent et al. (12) to be similar to that of elastin.

Myocardial tissue was homogenized in a 10-ml, all-glass Kontes tissue grind pestle with PBS at $4^{\circ} \mathrm{C}$. After the homogenate was centrifuged at $4,000 \mathrm{~g}$ for $10 \mathrm{~min}$, the supernatant, along with all subsequent supernatants, was retained so that the proteins could be recovered by precipitation in 5\% TCA; then the hydroxyproline content was determined. The residue was resuspended in $2 \%$ SDS and rehomogenized in the $10-\mathrm{ml}$ glass homogenizer at room temperature. The homogenate was again centrifuged and rehomogenized in $2 \%$ SDS; the procedure was repeated four times. The remaining residue was further extracted three times with PBS to remove the excess SDS. The residue was then rehomogenized in acetone and centrifuged at $4,000 \mathrm{~g}$ for $10 \mathrm{~min}$; then the supernatant was discarded. This step was repeated, and the pellet was dried under vacuum.

Digestion of collagen with cyanogen bromide. The acetone-dried powder was homogenized in an all-glass homogenizer with $0.6 \mathrm{ml}$ of $70 \% \mathrm{vol} / \mathrm{vol}$ of formic acid per $100 \mathrm{mg}$ of original tissue. The homogenate was transferred to a $15-\mathrm{ml}$ graduated polyethylene centrifuge tube, and any remaining residue was washed from the homogenizer with 0.6 $\mathrm{ml}$ of formic acid. The homogenate was made up to a volume of $1.5 \mathrm{ml}$ with $70 \% \mathrm{vol} / \mathrm{vol}$ formic acid per $100 \mathrm{mg}$ of original tissue. Cyanogen bromide crystals were added to this volume to produce a concentration of $20 \mathrm{mg} / \mathrm{ml}$. Nitrogen gas was then bubbled through the mixture, the tubes were sealed, and the reaction was allowed to proceed for $18 \mathrm{~h}$ at $25^{\circ} \mathrm{C}$. We used a digestion time of $18 \mathrm{~h}$ in our experiments rather than the 6-h digestion time recommended by Laurent et al. (12), because only $50 \%$ of the cardiac collagen was digested during $6 \mathrm{~h}$ but $80 \%$ was solubilized during $18 \mathrm{~h}$. To facilitate mixing, the tubes were positioned at an angle of $30^{\circ}$. When the reaction was completed, the digest was centrifuged at $5,000 \mathrm{~g}$ for $20 \mathrm{~min}$. The stopper was removed in a fume cupboard, and $0.3 \mathrm{ml}$ of the supernatant was removed and dried under vacuum in a $1.5-\mathrm{ml}$ microfuge tube. This was then directly dissolved in sample buffer in preparation for PAGE. The amount loaded was determined by hydroxyproline estimation on the lyophilized powder obtained from $0.5 \mathrm{ml}$ of supernatant. We used cyanogen bromide rather than pepsin for the extraction of collagen because $\mathrm{CNBr}$ digests $\sim 80 \%$ of cardiac collagen, but pepsin digested only $12 \%$ of the cardiac collagen in our studies. The percentage recovery of collagen in each step of extraction in terms of hydroxyproline is shown in Fig. 1.

\section{Collagen typing}

Gel electrophoresis. PAGE was performed on vertical gels (Protean II, Bio-Rad Laboratories, Inc., Richmond, CA) by stacking and separating gel concentrations of 4 and $12 \%$, respectively. The gels were $1.5 \mathrm{~mm}$ thick, with 15 sample wells. Samples of a volume of $10 \mu$ l were loaded into the wells using a microsyringe (Hamilton Co., Reno, NV), and stacking was allowed to occur at a current of $25 \mathrm{~mA}$. Once the samples entered the separating gel, the current was increased to $35 \mathrm{~mA}$, and electrophoresis continued until the dye marker reached a level two-thirds from the top. When electrophoresis was complete, the gel was removed and stained for $1 \mathrm{~h}$ by gentle shaking in $250 \mathrm{ml}$ of an aqueous solution containing $0.125 \%$ Coomassie blue $R 250,50 \%$ methanol, and $10 \%$ acetic acid. Using several changes of acetic acid, the gel was destained for $24-48 \mathrm{~h}$ by continuous shaking. All staining and destaining reactions, as well as gel buffers, were filtered to minimize contamination for subsequent scanning.

Gel scanning. Gels were scanned using Helena Laboratories Quick Scan. The heart collagen showed an electrophoretic pattern similar to that of collagen standards; the small degree of background staining made these gels suitable for densitometric scanning (Fig. 2). The quan-
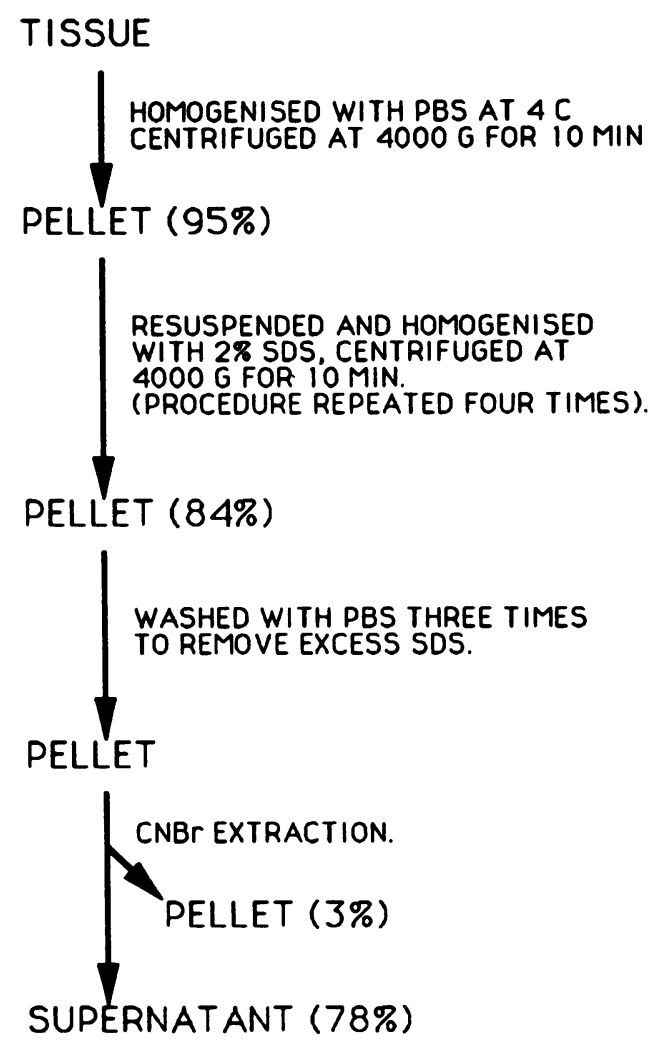

Figure 1. Percentage recovery of collagen during each step of cyanogen bromide $(\mathrm{CNBr})$ extraction, quantified by hydroxyproline estimation. 


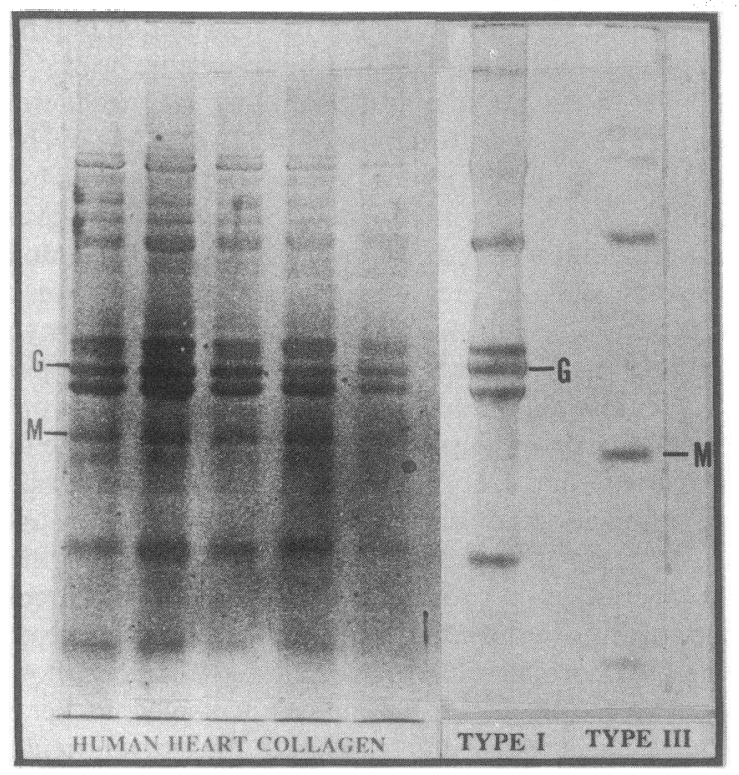

Figure 2. PAGE of $\mathrm{CNBr}$ digests of myocardial extracts and type I and III standards. The major bands used for quantification of collagen type I was band G, $\alpha_{1}$ (1) CB-8 and for type III band M, $\alpha_{1},(111)$ CB-5 plus $\alpha_{1}$ (111) CB-9.

titation of relative amounts of type I:III collagen was accomplished by determining the relationship between the amount of collagen applied to the gel and that found in the peak, a relationship that was distinctive for the collagen types. The major bands of type I G, $\left(\alpha_{1}[1]-\mathrm{CB}-8\right)$; band I, $\left(\alpha_{1}[1]-\mathrm{CB}-6\right)$; band B, $\alpha_{2}$ CB-3.5; as well as the major single band $\mathrm{M}$, $\alpha_{1}(111)$ CB-5; plus $\alpha_{1}$ (111)-CB-9 of type III gave good linear plots, with a correlation coefficient of $\sim 0.98$, as illustrated in Fig. 3. Band $G$ for type I collagen and $M$ for type III collagen were used for quantification of collagen phenotypes.

Quantitation collagen type I and type III. Four different concentrations of collagen standard I and III were applied in a gel, and a regression equation was obtained. The concentration of collagen type I and III in myocardial tissue was calculated from the regression equation of the standards.

\section{Statistical analysis}

We used an analysis of variance, and the values were corrected for age and sex. Individual group comparison was done using Bonferroni (Dunn) $t$ test analysis.

\section{Results}

Heart weights in cardiomyopathic patients and normal individuals. We studied three groups of patients, the first comprised of normal individuals who died of noncardiovascular causes (all deaths were due to motor vehicle accidents), the second of patients with ischemic cardiomyopathy who had not received captopril therapy, and the third of patients with ischemic cardiomyopathy who had received captopril therapy at a dose of $78.50 \pm 33.60 \mathrm{mg}$ for a duration of $2.22 \pm 0.27 \mathrm{yr}$. All patients with ischemic cardiomyopathy had a significantly increased heart weight of $487.03 \pm 104.73$ compared with that of $275.00 \pm 55.00$ in normal individuals. Patients with ischemic cardiomyopathy also had a significantly elevated heart weight/body weight ratio of $7.16 \pm 0.93$ compared with that of
$4.05 \pm 0.55$ in normal individuals. The results are summarized in Table $\mathrm{I}$.

Myocardial collagen concentration. The collagen concentration, defined by the amount of collagen in milligrams per gram of heart tissue estimated from the quantification of hydroxyproline, increased from $7.96 \pm 1.24 \mathrm{mg} / \mathrm{g}, n=6$ in normal myocardium to $13.97 \pm 1.30 \mathrm{mg} / \mathrm{g}, n=7, P<0.05$ in myocardium of patients with ischemic cardiomyopathy. Of these patients, those given captopril therapy before transplantation had a significantly lower left ventricular collagen concentration of $10.03 \pm 1.46 \mathrm{mg} / \mathrm{g}, P<0.05$ compared with that in patients receiving no captopril. Moreover, the collagen concentration of the patients given captopril did not significantly differ from that seen in the normal myocardium when the results were corrected for age (Table II).

Distribution of collagen phenotypes in cardiomyopathic patients and normal individuals. The collagen type I and type III concentration was calculated from quantification of the cyanogen bromide fragments representative of each type. Cyanogen bromide digested $\sim 80 \%$ of cardiac collagen, and the percentage of collagen recovery in each step of extraction determined by hydroxyproline estimation is shown in Fig. 1. Repeated homogenization with SDS removed most of the noncollagen proteins, with very little loss of collagen in the supernatant. The pellet obtained after homogenization with SDS contained $\sim 84 \%$ of the collagen present in the original tissue sample, and $\mathrm{CNBr}$ digested almost all of this collagen.

To validate our quantification procedure, we loaded three lanes of the gel, the first with $10 \mu \mathrm{g}$ standard, the second with 10 $\mu \mathrm{g}$ standard plus $10 \mu \mathrm{g}$ myocardial collagen, and the third with $10 \mu \mathrm{g}$ myocardial collagen. In these experiments, the percent-

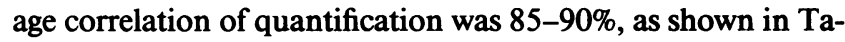
ble III.

We detected no statistical difference in the type I collagen concentration of $4.94 \pm 1.28 \mathrm{mg} / \mathrm{g}$ in normal individuals and that of $6.78 \pm 0.99$ in patients with ischemic cardiomyopathy

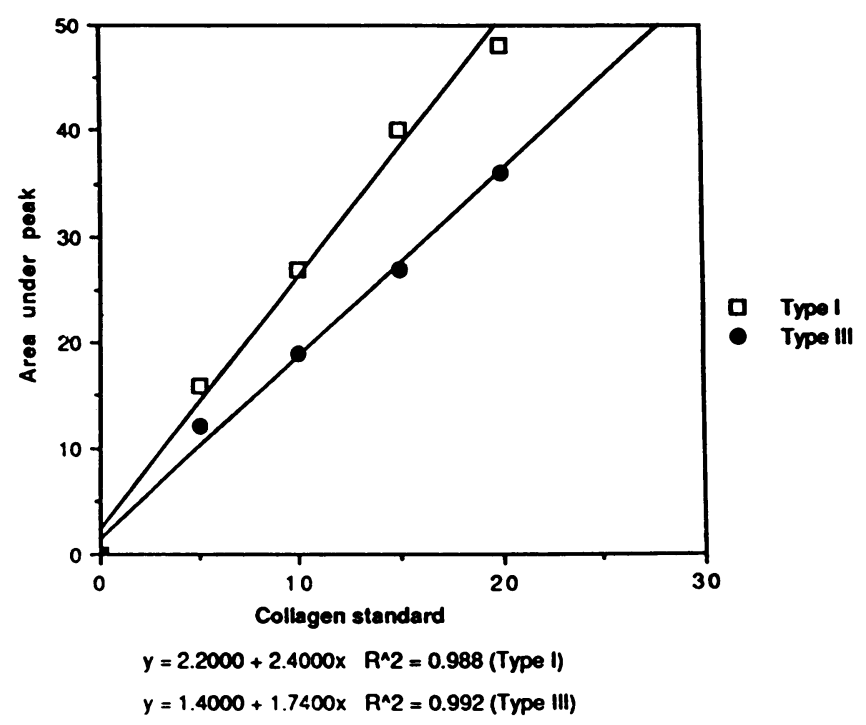

Figure 3. Relationship between peak area and collagen loaded onto the gel for type I collagen and type III collagen. 5, 10, 15, and $20 \mathrm{mg}$ of collagen standards were loaded in a SDS-polyacrylamide gel. Band G, $\left(\alpha_{1}[1]-C B-8\right)$ for type I and band M, $\alpha_{1}(111)$ CB-5 plus $\alpha_{1}(111)$ CB-9 for type III were used for quantification of specific types. 
Table I. Patient Characteristics

\begin{tabular}{cccc}
\hline \multicolumn{1}{c}{ Patients } & Age & Heart wt & Heart wt/body wt \\
\hline & $y r$ & $g$ & $g / k g$ \\
$\begin{array}{l}\text { Normal } \\
(n=6)\end{array}$ & $32.00 \pm 16.40$ & $275.00 \pm 55.0$ & $4.05 \pm 0.55$ \\
$\begin{array}{l}\text { Ischemic } \\
\mathrm{CM}(n=7)\end{array}$ & $49.71 \pm 3.49$ & $474.57 \pm 109.58$ & $6.81 \pm 0.81$ \\
$\begin{array}{l}\text { Ischemic CM } \\
\text { received } \\
\text { captopril* } \\
(n=7)\end{array}$ & $54.28 \pm 7.15$ & $500.08 \pm 98.56$ & $7.52 \pm 1.04$ \\
\hline
\end{tabular}

* Received captopril for a duration of $2.22 \pm 0.27$ yrs at a dose of $78.50 \pm 33.60 \mathrm{mg}$.

(all 14 patients). Moreover, we detected no significant difference in the type I collagen concentration of $7.29 \pm 0.73 \mathrm{mg} / \mathrm{g}$ in patients receiving no captopril $n=7$ and that of $6.29 \pm 1.20$ $\mathrm{mg} / \mathrm{g}, n=7$ in those who did. The type III collagen concentration increased from the normal value of $2.56 \pm 0.21 \mathrm{mg} / \mathrm{g}$ to $5.51 \pm 0.39 \mathrm{mg} / \mathrm{g}, P<0.05$ (all 14 patients), the value found in patients with ischemic cardiomyopathy. In patients with ischemic cardiomyopathy receiving captopril therapy, the collagen type III concentration was $4.87 \pm 0.64 \mathrm{mg} / \mathrm{g}, n=7$, a value significantly lower than that of $6.10 \pm 0.58 \mathrm{mg} / \mathrm{g}, P<0.05, n=7$ in patients receiving no captopril therapy. However, even in those receiving captopril, this figure was significantly higher than that seen in individuals with normal myocardium. The collagen type I:III ratio decreased significantly from $1.93 \pm 0.52$, $n=6$ in normal myocardium to $1.23 \pm 0.27, n=7, P<0.05$, a value seen in the myocardium of patients with ischemic cardiomyopathy. Patients given captopril therapy had a type I:III ratio of $1.49 \pm 0.38$, which did not differ significantly from that seen in normal myocardium (Table II).

\section{Discussion}

In this study we have shown that an increased collagen concentration exists in the myocardium of patients with ischemic cardiomyopathy, and that this increase is associated with an increase in type III collagen. We have also shown that treatment with captopril can partially normalize the distribution of types I and III collagen in this ischemic cardiomyopathic heart.

That collagen plays a role in determining the mechanical properties of the heart is well established (4). In a review, Weber reiterated (5) that the hypertrophic process is accompanied by a pressure overload inducing the increase of collagen synthesis, fibroblast proliferation, and structural and biochemical remodeling of the matrix. This increase also includes the formation of myocardial fibrosis, which alters the diastolic and systolic myocardial stiffness that may eventually lead to pathologic hypertrophy. Weber also suggested that a loss of collagen titers or a decline in the matrix of tensile strength can be responsible for both the regional and global transformations in myocardial architecture and function seen in stunned myocardium and dilated cardiomyopathy (5). Jugdutt et al. (13) have shown that over a 6-wk period after myocardial infarction occurs, the collagen concentration in the infarcted segment rises progressively to five times its normal value. In noninfarcted regions, however, the collagen concentration remains unchanged. Pfeffer et al. (14) found a progressive enlargement in the left ventricular chamber size and the filling volume $1 \mathrm{yr}$ after an inferior myocardial infarction had occurred. All mechanisms responsible for this progressive dilatation remain unknown. Furthermore, after reviewing studies on collagen and its relationship to myocardial function, Weber (5) concluded that the amount of collagen present in the heart is not the sole factor responsible for the cardiac stiffness but that the structural characteristics and the arrangement of the collagen fiber with respect to myocyte is the major factor influencing the mechanical behavior of the heart. The data presented in this study also support this conclusion.

In this study we have shown that there is an increase in collagen concentration in ischemic cardiomyopathic hearts compared with that in normal hearts. Patients with ischemic cardiomyopathy receiving captopril therapy exhibited a considerable lowering of the collagen concentration compared with that in untreated patients. In these untreated patients, the interval of time between the onset of disease and its progression to transplantation was $9.85 \pm 8.25 \mathrm{mo}$, one of the intriguing observations of this study. On the other hand, the time interval for patients treated with captopril was considerably longer, $42.40 \pm 26.29 \mathrm{mo}$. A comparison of these time intervals suggests that captopril therapy resulting in the reduction of collagen concentration may play a role in prolonging the time from the

Table II. Total Collagen Concentration and Collagen Type I and Type III in Ischemic Cardiomyopathy and Effect of Captopril Therapy

\begin{tabular}{llcccc}
\hline & $\begin{array}{c}\text { Normal } \\
(n=6)\end{array}$ & $\begin{array}{c}\text { Ischemic CM } \\
(n=7)\end{array}$ & $\begin{array}{c}\text { Ischemic CM } \\
\text { received captopril } \\
(n=7)\end{array}$ & $\begin{array}{c}\text { Normal } \\
\text { vs. } \\
\text { ischemic CM }\end{array}$ & $\begin{array}{c}\text { Ischemic CM } \\
\text { vs. } \\
\text { ischemic CM } \\
\text { (captopril) }\end{array}$ \\
\hline $\begin{array}{c}\text { Collagen } \\
\text { concentration } \\
(m g / g)\end{array}$ & $7.96 \pm 1.24$ & $13.97 \pm 1.30$ & $10.03 \pm 1.46$ & $P<0.05$ & $P<0.05$ \\
$\begin{array}{c}\text { Normal } \\
\text { vs. } \\
\text { ischemic CM } \\
\text { (captopril) }\end{array}$ \\
$\begin{array}{c}\text { Collagen I:III } \\
\text { ratio }\end{array}$ & $1.93 \pm 0.52$ & $1.23 \pm 0.27$ & $1.49 \pm 0.38$ & $P<0.05$ & NS \\
$\begin{array}{c}\text { Collagen type I } \\
(m g / g)\end{array}$ & $4.94 \pm 1.28$ & $7.29 \pm 0.73$ & $6.29 \pm 1.20$ & NS & NS \\
$\begin{array}{c}\text { Collagen type III } \\
(m g / g)\end{array}$ & $2.56 \pm 0.21$ & $6.10 \pm 0.58$ & $4.87 \pm 0.64$ & $P<0.05$ & $P<0.05$ \\
\hline
\end{tabular}


Table III. Quantification of Collagen

\begin{tabular}{lcccc}
\hline & \multicolumn{3}{c}{ Area under peak } \\
\cline { 2 - 4 } Collagen & $10 \mu \mathrm{g} \mathrm{CS}$ & $\begin{array}{c}10 \mu \mathrm{g} \mathrm{CS} \\
+10 \mu \mathrm{g} \mathrm{HC}\end{array}$ & $10 \mu \mathrm{g} \mathrm{HC}$ & $\begin{array}{c}\text { Predicted } \\
\text { \% correlation }\end{array}$ \\
\hline Type I & 27.00 & 44.00 & 19.00 & 90.00 \\
Type III & 19.00 & 35.00 & 19.00 & 88.00 \\
\hline
\end{tabular}

HC, heart collagen; CS, collagen standard.

onset of the disease to the transplantation of the heart. But the progress of the disease may also be associated with a continuous loss of myocytes, leading to deteriorated myocardial function and ultimately to the need for transplantation.

We have found a significant decrease in the ratio of type I:III collagen in patients with ischemic cardiomyopathy. In those receiving captopril, the ratio of collagen type I:III does not significantly differ from that in normal individuals. We found the distribution of collagen type I concentration to be similar in all three groups studied, in normal individuals, patients with ischemic cardiomyopathy, and ischemic cardiomyopathic patients treated with captopril therapy. Although patients receiving captopril showed some reduction in type III collagen, this value is still significantly higher than that in normal controls. In fact, when treated and untreated patients were pooled and their patient data grouped, the type III collagen value, $5.51 \pm 0.39$, is significantly greater than that of $2.56 \pm 0.21$ $\mathrm{mg} / \mathrm{g}, P<0.05$ in normal individuals. Therefore, our study confirmed that compared with normal individuals, patients with ischemic cardiomyopathy showed a significant increase in type III collagen, although this increment was only partly reduced after they had received captopril. This data suggest that the collagen type I remains unchanged during the progression of the disease, but a significant increase in type III collagen concentration is found in all patients with ischemic cardiomyopathy. Thus, the accumulation of type III compared with type I collagen may play a role in determining the myocardial stiffness in ischemic cardiomyopathy (4). The increased deposition of type III collagen may also be a reaction to other abnormalities rather than a casual factor itself.

This is the first demonstration that treatment with antihypertensive drugs can alter the phenotypic distribution of collagen in humans, although the patients in our study did not show a significant change in collagen type I concentration. Shekhonin et al. (10) showed a diffuse distribution of collagen type III in postinfarcted cardiosclerosis and observed that type I collagen occurs mainly in the deeper regions of fibrous tissue. The selective increase regarding collagen type III found in our study may be an important marker leading to myocardial dysfunction in patients with ischemic cardiomyopathy. Our study shows that the type of collagen present rather than the total amount of collagen deposited may be a key factor in determining the myocardial function.

In the rodent model we had reported a similar observation (6) in SHR. In this model we found an increase in type I collagen deposition during the compensated myocardial hypertrophy and, in older SHR about 18 mo of age, a marked increase in type III collagen deposition as the cardiac function deteriorated. Furthermore, we have also shown in this model that treatment with captopril for 6 wk causes not only a signifi- cant reduction in collagen content of the heart but also a normalization of type I:III. Therefore, these reports (6) in the SHR hypertensive hypertrophy model seem similar to the observations in our present study of humans.

One of the intriguing observations we found in SHR during the course of the development of hypertrophy in hypertension was that the ratio of type I:III collagen phenotypes is not constant throughout the evolution of the disease process. During the early phases of hypertrophy (6 mo), the type I collagen was more prevalent. In fact, the total collagen was composed primarily of type I, which is associated with the improved functional ability of the heart, defined by measuring both positive and negative $d p / d t$ (unpublished observation). On the other hand, as the disease progressed (18 mo of age), the type III collagen increased and the cardiac function deteriorated. During the course of development of human hypertrophy, especially concentric hypertrophy, the disease process may be associated with an increased deposition of type I collagen, an occurrence also seen in 34-wk-old SHR. When the decomposition in human myocardium begins, the accompanying dilation of the left ventricle may be associated with a marked increase in type III collagen. This is the course of events we had observed in older SHR (6). In ischemic cardiomyopathy, the presence of a large proportion of this thin type III collagen fiber, known to have less tensile strength than the thicker type I fiber, is perhaps responsible for the dilatation of the myocardium that may result in the spherical formation of the left ventricular chamber. If this course of events is accurate, an increased formation of thick type I fibers would be found in the myocardium of patients with hypertrophic cardiomyopathy. We are now studying the collagen phenotype distribution in these patients.

The mechanism by which the collagen content becomes increased in the cardiomyopathic heart has not yet been identified, although it may involve hypoxia (15) produced by diffuse cardiosclerosis resulting from atherosclerosis of the coronary artery. The initial increase in collagen deposition would lead to further hypoxia, setting off a cycle leading to severe myocardial dysfunction. Sen et al. (16) have shown that different drugs effect collagen metabolism differently. Their study showed that captopril reduces the collagen content of the myocardium, that hydralazine has no effect on collagen content, and that treatment with alpha-methyldopa actually increases collagen content. These results may partly explain why captopril therapy has some beneficial effects in treating cardiovascular disease. To date, captopril was found to be the only drug to reduce myocardial collagen in rodent models, although its action may be due to an improvement in the hemodynamic status of the heart. Alternatively, captopril may somehow directly affect the biosynthetic pathway of myocardial collagen production, or a combination of both alternatives may be at work. The exact mechanism by which captopril alters collagen metabolism remains to be elucidated. The present study demonstrates that collagen phenotypic distribution is partially altered as a result of captopril therapy, and that this alteration occurs independently of that of myocardial mass, because treatment with captopril did not regress cardiac hypertrophy, an event observed in the rodent model (6). This might suggest that the connective tissue metabolism and myocyte growth in ischemic cardiomyopathy are regulated by two separate mechanisms. Further studies are necessary to define the mechanism by which the collagen synthesis is regulated and how the regression of hypertrophy is achieved by antihypertensive therapy. 


\section{Acknowledgments}

We gratefully acknowledge the help of Amy Rogers, R.N., and Marsha Lowrie, R.N., in providing patient information. We also express our gratitude to Dr. Stewart and members of the transplant team who helped us obtain the myocardial tissues. We thank Laura Battista for typing the manuscript and Suzanne Hazan for editorial assistance.

This study was supported in part from National Institutes of Health (NIH) grant RO-1-HL-27838. Statistical analysis was done using the PROPHET computer system, which is supported in part by NIH. Debabrata Mukherjee is an American Heart Association Fellow.

\section{References}

1. Burch, G. E., T. D. Criles, and H. L. Colcolough. 1970. Ischemic cardiomyopathy. Am. Heart J. 79:291-292.

2. Burch, G. E., and C. D. McDonald. 1971. Prolonged bed rest in the treatment of ischemic cardiomyopathy. Chest. 60:424-430.

3. Burch, G. E., G. Y. Tsui, and J. M. Harb. 1972. Ischemic cardiomyopathy. Am. Heart J. 83:340-350.

4. Medugorac, I., and R. Jacob. 1983. Characterization of left ventricular collagen in the rat. Cardiovasc. Res. 17:15-21.

5. Weber, K. T. 1989. Cardiac interstitium in health and disease: the fibrillar collagen network. J. Am. Coll. Cardiol. 13:1636-1652.

6. Mukherjee, D., and S. Sen. 1990. Collagen phenotypes during development and regression of myocardial hypertrophy in spontaneously hypertensive rats. Circ. Res. 67:1474-1480.
7. Hurst, J. W., editor. 1990. The Heart Arteries and Veins. McGraw-Hill Book Co., New York. 1090 pp.

8. Faxon, D. P. 1988. ACE inhibitions for the failing heart. Experiments with captopril. Am. Heart J. 115:1085-1093.

9. Epstein, E. M., and N. H. Munderloh. 1975. Isolation and characterization of $\mathrm{CNBr}$ peptides of human $[\alpha 1(\mathrm{III})]_{3}$ collagen and tissue distribution of $[\alpha 1(\mathrm{I})]_{2} \alpha 2$ and $[\alpha 1(\mathrm{III})]_{3}$ collagens. J. Biol. Chem. 250:9304-9312.

10. Shekhonin, B. V., S. P. Domogatsky, G. L. Idelson, and V. E. Kotelianskyh. 1988. Participance of fibronectin and various collagen types in the formation of fibrous extracellular matrix in cardiosclerosis. J. Mol. Cell. Cardiol. 20:501-508.

11. Bergman, I., and R. Loxley. 1963. Two improved and simplified methods for the spectrophotometric determination of hydroxyproline. Anal. Chem. 35:1961-1965.

12. Laurent, G. J., P. Cockerill, R. J. McAnulty, and J. R. B. Hastings. 1981. A simplified method for quantification of the relative amounts of type I and type III collagen in small tissue samples. Anal. Biochem. 113:301-312.

13. Jugdutt, B. I., and R. W. M. Amy. 1986. Healing after myocardial infarction in the dog: changes in infarct hydroxyproline and topography. J. Am. Coll. Cardiol. 7:91-102.

14. Pfeffer, M. A., G. A. Lamos, D. Vaughn, F. Parisi, and E. Braunwald. 1988. Effect of captopril on progressive ventricular dilatation after interior myocardial infarction. N. Engl. J. Med. 391:80-86.

15. Bartosova, D., M. Chapil, B. Korecky, O. Poupa, K. Rakusan, Z. Turek, and M. Vizek. 1969. The growth of the muscular and collagenous parts of the rat heart in various forms of cardiomegaly. J. Physiol. (Lond.). 200:285-295.

16. Sen, S., and F. M. Bumpus. 1979. Collagen synthesis in development of reversal of cardiac hypertrophy in spontaneously hypertensive rats. Am. J. Cardiol. 44:954-958. 